\title{
ROCK2 mediates the proliferation of pulmonary arterial endothelial cells induced by hypoxia in the development of pulmonary arterial hypertension
}

\author{
FENG QIAO $^{1}$, ZHITIAN ZOU ${ }^{1}$, CHUNHUI LIU ${ }^{1}$, XIAOFENG ZHU $^{1}$, XIAOQIANG WANG $^{1}$, \\ CHENGPENG YANG ${ }^{1}$, TENGJIAO JIANG ${ }^{1}$ and YING CHEN $^{2}$ \\ Departments of ${ }^{1}$ Thoracic Surgery and ${ }^{2}$ Intensive Care Unit, The First Affiliated Hospital of Jiamusi University, \\ Jiamusi, Heilongjiang 154002, P.R. China
}

Received February 1, 2015; Accepted March 10, 2016

DOI: $10.3892 / \mathrm{etm} .2016 .3214$

\begin{abstract}
It has been reported that RhoA activation and Rho-kinase (ROCK) expression are increased in chronic hypoxic lungs, and the long-term inhibition of ROCK markedly improves the survival of patients with pulmonary arterial hypertension (PAH). However, whether Rho-kinase $\alpha$ (ROCK2) participates in regulation of the growth of pulmonary arterial endothelial cells (PAECs) remains unknown. The aim of the present study was to investigate the effect of hypoxia on the proliferation of PAECs and the role of ROCK2 in the underlying mechanism. The results of western blotting and reverse transcription-quantitative polymerase chain reaction analysis showed that hypoxia increased the activity and expression of ROCK2 in PAECs, and the stimulating effects of hypoxia on the proliferation of PAECs were attenuated by either the ROCK inhibitor Y27632 or transfection with ROCK2 small interfering RNA. Moreover, analysis of cyclin A and cyclin D1 mRNA expression indicated that ROCK2 mediates the cell cycle progression promoted by hypoxia. These results indicate that hypoxia promotes the proliferation of pulmonary arterial endothelial cells via activation of the ROCK 2 signaling pathway.
\end{abstract}

\section{Introduction}

Pulmonary arterial hypertension (PAH) is a fatal condition characterized by increased pulmonary vascular resistance and finally leading to right heart failure and mortality $(1,2)$. Endothelial injury, prolonged vasoconstriction and the

Correspondence to: Dr Ying Chen, Department of Intensive Care Unit, The First Affiliated Hospital of Jiamusi University, 348 Dexiang Road, Xiangyang, Jiamusi, Heilongjiang 154002, P.R. China

E-mail: yingchenjms@126.com

Key words: hypoxia, pulmonary arterial endothelial cells, proliferation, ROCK2, pulmonary arterial hypertension proliferation and migration of vascular smooth muscle cells (VSMCs), are causes of increased pulmonary vascular resistance (3). Multiple pharmacological agents, such as vasodilators and anticoagulants, have been developed for the treatment of PAH; however, the long-term prognosis of patients with severe PAH remains poor (3). Therefore, there is an urgent requirement for the development of more effective treatments for PAH.

Rho-kinase (ROCK) is a member of the serine/threonine kinase family that is an important downstream effector of the small GTP-binding protein RhoA. The Rho/ROCK pathway plays an important role in various fundamental cellular functions, including contraction, motility, proliferation and migration $(4,5)$. There are two isoforms of ROCK, namely ROCK1 (Rho-kinase $\beta$ ) and ROCK2 (Rho-kinase $\alpha$ ) (6). ROCK1 and ROCK 2 are highly homologous with regard to amino acid sequence and kinase domains, sharing $~ 65 \%$ homology in amino acid sequence and $92 \%$ homology in their kinase domains (6). Although the two isoforms are ubiquitously expressed in invertebrates and vertebrates, ROCK1 is expressed mainly in circulating inflammatory cells and ROCK2 is expressed in vascular cells $(7,8)$. Homozygous ROCK1-deficient mice show open eyelids at birth and omphalocele, whereas homozygous ROCK2-deficient mice die embryonically because of placental dysfunction, suggesting that ROCK1 and ROCK 2 mediate different functions in different types of cells $(9,10)$. To date, to the best of our knowledge, whether ROCK is responsible for the growth of pulmonary arterial endothelial cells (PAECs) has not yet been evaluated.

The present study was conducted to investigate the effect of hypoxia on the proliferation of pulmonary arterial endothelial cells (PAECs) and the role of ROCK2 in the underlying mechanism. The activity and expression of ROCK2 were evaluated in PAECs under hypoxic conditions, and the growth and proliferation of PAECs were evaluated. In addition, the effects of hypoxia on the expression of cyclin D and cyclin A, and the attenuating effects of either a ROCK inhibitor or ROCK2 small interfering RNA (siRNA) were tested. The results may reveal an important underlying mechanism of PAEC overgrowth in the progression of PAH. 


\section{Materials and methods}

Materials. The Y27632 ROCK inhibitor was purchased from Cayman Chemical Co. (Ann Arbor, MI, USA). Rabbit anti-myosin phosphatase target subunit 1 (anti-MYPT1; 2634) and anti-phospho (p)-MYPT1 (4563) polyclonal antibodies, and horseradish peroxidase (HRP)-conjugated goat anti-rabbit IgG (7074) and anti-mouse $\operatorname{IgG}$ (7076) secondary antibodies were purchased from Cell Signaling Technology, Inc. (Danvers, MA, USA). Antibodies against rabbit polyclonal ROCK2 (BA1766) and mouse monoclonal $\beta$-actin (BM0626) were purchased from Boster Wuhan Biological Technology, Co., Ltd. (Wuhan, China). 5-Bromo-2'-deoxyuridine (BrdU) proliferation assay kit was from EMD Millipore (Billerica, MA, USA). All other reagents were from common commercial sources.

Cell preparation and culture. PAECs were isolated from fresh bovine pulmonary tissues as previously described (11). The bovine tissues were obtained from a local slaughterhouse with all protocols reviewed and approved by the Ethics Committee of Laboratory Animals at Jiamusi University (Jiamusi, China). The identity was confirmed by typical endothelial cell morphology and by positive anti-factor VIII staining. The cells were cultured with Dulbecco's modified Eagle's medium (DMEM) supplemented with 20\% fetal bovine serum (FBS; both Thermo Fisher Scientific, Inc., Waltham, MA, USA) in a $37^{\circ} \mathrm{C}, 5 \% \mathrm{CO}_{2}$ humidified incubator. Passages 2-5 were used for further experimentation.

Small interfering RNA (siRNA) design and transfections. PAECs were transfected with ROCK2 siRNA (siROCK2), which was designed and synthesized by Shanghai GenePharma Co., Ltd. (Shanghai, China). Non-targeted control siRNA (siNC) was used as a negative control. The transfection protocol was that PAECs were cultured until $30-50 \%$ confluence and then $1.5 \mu \mathrm{g}$ siRNAs and $7.5 \mu \mathrm{l} \mathrm{X}$-treme Gene transfection reagent (Roche Diagnostics, Shanghai, China) were diluted in serum-free Opti-MEM-1 medium (Thermo Fisher Scientific, Inc.), respectively. The siRNAs and transfection reagent were gently mixed together. After incubating at room temperature for $20 \mathrm{~min}$, the mixture was added directly onto the cells. Following transfection, cells were quiesced in DMEM for $24 \mathrm{~h}$ and used as required.

Cell treatment and groups. Firstly, cells were divided into three groups: Normoxia $\left(20 \% \mathrm{O}_{2}\right)$, hypoxia $\left(5 \% \mathrm{O}_{2}\right)$ and hypoxia plus $\mathrm{Y} 27632\left(5 \% \mathrm{O}_{2}+1 \mu \mathrm{M}\right.$ Y27632). Following 24-h treatment, protein and RNA were extracted from these cells. Secondly, cells were also divided into three groups: Normoxia + siControl, hypoxia + siControl and hypoxia + siROCK2. Following 24-treatment, protein and RNA were extracted from these cells.

3-(4,5-Dimethylthiazol-2-yl)-2,5-diphenyl-tetrazolium bromide (MTT) assay. PAECs were cultured at a density of $\sim 1 \times 10^{4}$ per well in 96-well culture clusters, and then the cells were treated with indicated reagents as groups. Dimethyl sulfoxide (DMSO) and other agents at the indicated concentrations were added for $24 \mathrm{~h}$. Afterwards, the cells were incubated with
$0.5 \%$ MTT, which is a yellow mitochondrial dye, in sterile phosphate-buffered saline for $4 \mathrm{~h}$ at $37^{\circ} \mathrm{C}$. The reaction was terminated by incubating the cells with DMSO for $10 \mathrm{~min}$. The absorbance at $540 \mathrm{~nm}$ was measured using an Epoch 2 spectrophotometer (BioTek China, Beijing, China). The amount of blue formazan dye, formed from MTT, is proportional to the number of surviving cells.

BrdU incorporation. PAECs were plated at $1 \times 10^{4}$ cells/well in 96-well plates, and then subjected to growth arrest for $24 \mathrm{~h}$ by replacing the DMEM plus $1 \%$ FBS with DMEM prior to various treatments. BrdU incorporation was measured using a BrdU proliferation assay kit according to the manufacturer's protocol. Briefly, the cells were labeled with $10 \mathrm{ng} / \mathrm{ml} \mathrm{BrdU}$ during the incubation, washed 3 times with cold wash buffer, fixed, air-dried and incubated $1 \mathrm{~h}$ at room temperature with mouse anti-BrdU monoclonal antibody (1:200). The antibody was aspirated, the cells were washed three times and then incubated with HRP-conjugated goat anti-mouse $\operatorname{IgG}$ $(1: 2,000)$ at room temperature for $30 \mathrm{~min}$. The cells were washed 3 times, and $100 \mu 1$ substrate was added to each well and incubated for $30 \mathrm{~min}$ in the dark. Thereafter, the absorbance measured at dual-wave lengths between 450 and $540 \mathrm{~nm}$ was determined.

Western blot analysis. Proteins from different experimental groups were solubilized and extracted as previously reported (12). The protein concentrations were determined by bicinchoninic acid protein assay (Pierce Biotechnology, Inc., Rockford, IL, USA) with bovine serum albumin (Thermo Fisher Scientific, Inc.) as a standard. Equal amounts of protein $(20 \mu \mathrm{g})$ from each sample were subjected to electrophoresis on a $10 \%$ sodium dodecyl sulfate-polyacrylamide gel, and transferred onto a nitrocellulose membrane (EMD Millipore). The blots were then incubated in a blocking buffer (Tris $20 \mathrm{mM}$, pH 7.6, $\mathrm{NaCl} 150 \mathrm{mM}$, and Tween $200.1 \%$ ) containing 5\% nonfat dry milk powder for $1 \mathrm{~h}$ at room temperature. This was followed by incubation with anti-MYPT1 $(1: 1,000)$, anti-p-MYPT1 (1:1,000), anti-ROCK2 (1:250) and $\beta$-actin (1:500) primary antibodies overnight at $4^{\circ} \mathrm{C}$, and incubation with HRP-conjugated goat anti-rabbit IgG and anti-mouse IgG secondary antibodies (both 1:3,000) at room temperature and enhanced chemiluminescence reagent (Thermo Fisher Scientific, Inc.) the next day. $\beta$-actin was used as the internal control in all experiments.

Reverse transcription-quantitative polymerase chain reaction (RT-qPCR). Total RNA was extracted using TRIzol (Invitrogen; Thermo Fisher Scientific, Inc.) and was reverse transcribed into cDNA using a PrimeScript RT kit (Takara Biotechnology Co., Ltd., Dalian, China). Reverse transcription reaction mixture contained added $2 \mu 15 \mathrm{X}$ PrimeScript buffer, $0.5 \mu \mathrm{l}$ PrimeScript RT enzyme mix I, $0.5 \mu \mathrm{l}$ oligo dT Primer $(50 \mu \mathrm{M}), 0.5 \mu 1$ random hexamers $(100 \mu \mathrm{M})$, $500 \mathrm{ng}$ RNA and RNase-free $\mathrm{dH} 2 \mathrm{O}$ to $10 \mu \mathrm{l}$, and was performed at $37^{\circ} \mathrm{C}$ for $15 \mathrm{~min}$ and $85^{\circ} \mathrm{C}$ for $5 \mathrm{sec}$, followed by holding at $4^{\circ} \mathrm{C}$. Applied Biosystems (ABI) 7300 Fast Real-Time PCR system (Thermo Fisher Scientific, Inc.) was used to perform qPCR experiments. Applied Biosystems Primer Express 3.0 (Thermo Fisher Scientific, Inc.) was used 
A

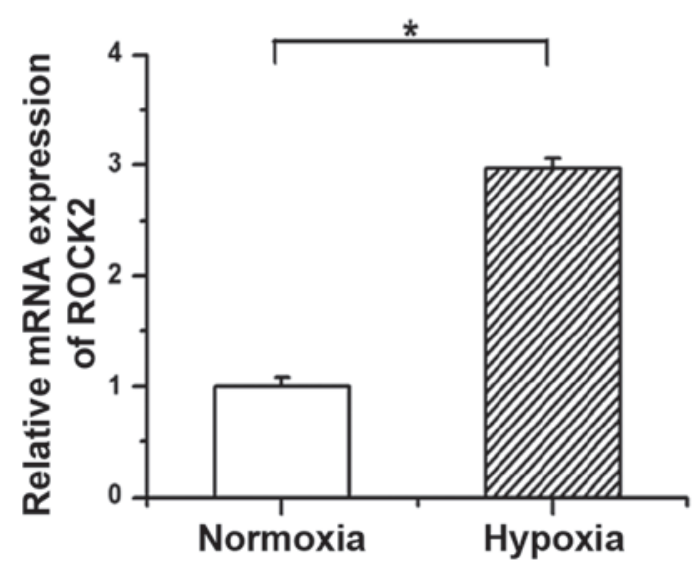

B

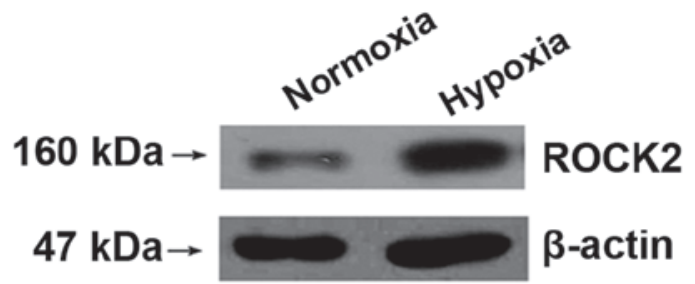

C

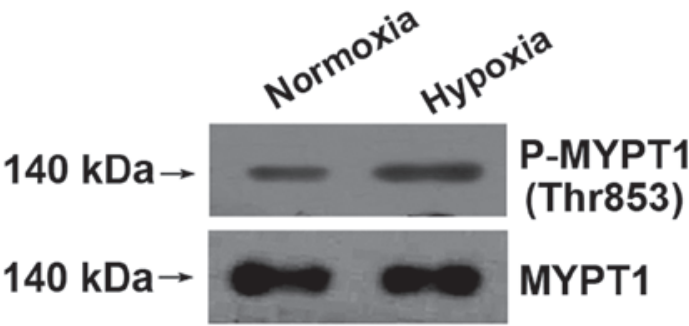

Figure 1. Hypoxia increases the activity and expression of ROCK2 in pulmonary arterial endothelial cells. (A) Treatment with hypoxia induced the mRNA expression of ROCK2 in PAECs. (B) The protein expression of ROCK2 was increased under conditions of hypoxia. (C) The phosphorylation of MYPT1 was significantly increased in PAECs following stimulation with hypoxia. Values are represented as mean \pm standard error of the mean from three or more independent batches of cells. "P $<0.05$, as detected by Student's t-test. ROCK2, Rho-kinase $\alpha$; MYPT1, myosin phosphatase target subunit 1; P-MYPT1, phospho-MYPT1.
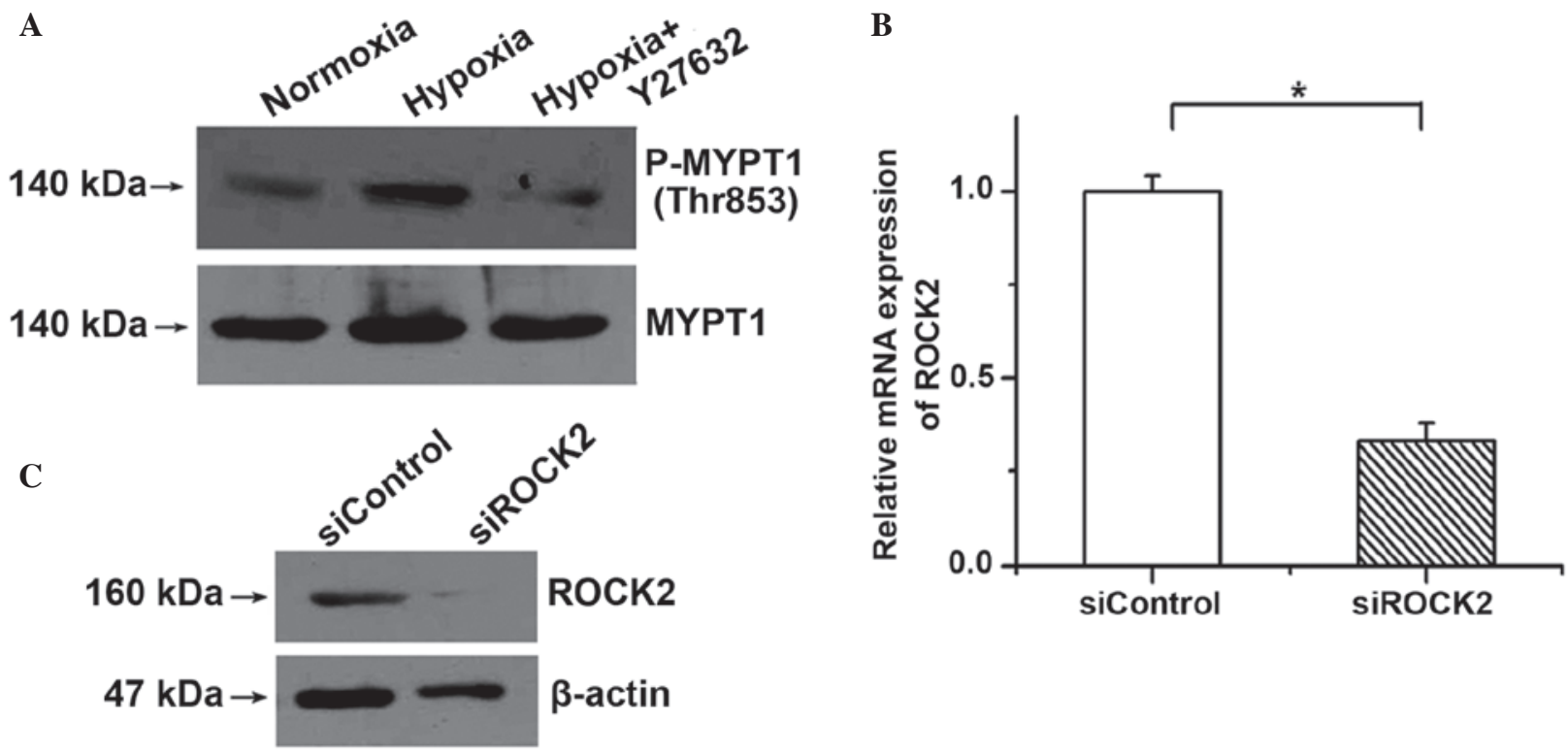

Figure 2. ROCK2 activity is reduced by Y27632, and ROCK2 siRNA (siROCK2) significantly knocks down the expression of ROCK2 in pulmonary arterial endothelial cells. (A) The phosphorylation of MYPT1 induced by hypoxia was clearly decreased by incubation with Y27632. (B) The mRNA expression of ROCK2 was knocked down by siROCK2. (C) The protein expression of ROCK 2 was repressed by siROCK2. Values are represented as mean \pm standard error of the mean from three or more independent batches of cells. "P<0.05, as detected by Student's t-test. ROCK2, Rho-kinase $\alpha$; siRNA, small interfering RNA; siControl, control siRNA; MYPT1, myosin phosphatase target subunit 1; P-MYPT1, phospho-MYPT1.

to design specific primers and BLAST analysis was used to confirm the specificity of the primers. Each $20-\mu 1$ reaction contained $10 \mu \mathrm{M}$ forward and reverse primers, $1 \mathrm{X} \mathrm{SYBR}^{\circledR}$ Premix Ex Taq ${ }^{\mathrm{TM}}$ II, $0.4 \mu \mathrm{l}$ ROX reference dye (both Thermo Fisher Scientific, Inc.) and $2 \mu \mathrm{l}$ cDNA. The ABI 7300 system was programmed with the PCR conditions: $95^{\circ} \mathrm{C}$ for $30 \mathrm{sec}$, 40 cycles of $95^{\circ} \mathrm{C}$ for $5 \mathrm{sec}$, and $60^{\circ} \mathrm{C}$ for $30 \mathrm{sec}$, and this was followed by routine melting curve analysis. Primer sequences were as follows: ROCK2, forward 5'-CTAGGC CGGGCGAAGC-3' and reverse 5'-CTCCAGCTTCCTCTG ACGAC-3'; cyclin D1, forward 5'-TCAAGTGTGACCCGG
ACTG-3' and reverse 5'-AAGCCAGACCAGCTTCTTCC-3'; cyclin A, foward 5'-GCT GTG CGT TGC GGTTC-3' and reverse 5'-GTGCGACTCCACTCT TCGAG-3'; $\beta$-actin, forward 5'-AGGCCCCTCTGAACCCTAAG-3' and reverse 5'-CCAGAGGCATACAGGGACAAC-3'. The target gene expression relative quantitation (RQ) was calculated by the $2{ }^{-\Delta \Delta \mathrm{Cq}}$ method (13). The first step in the RQ analysis was to normalize the target gene expression level to $\beta$-actin $(\Delta \mathrm{Ct})$ and the second step was to compare the difference in normalized target gene expressions between different samples $(\Delta \Delta \mathrm{Ct})$. Each experiment was repeated 2-3 times in 3-4 samples. 

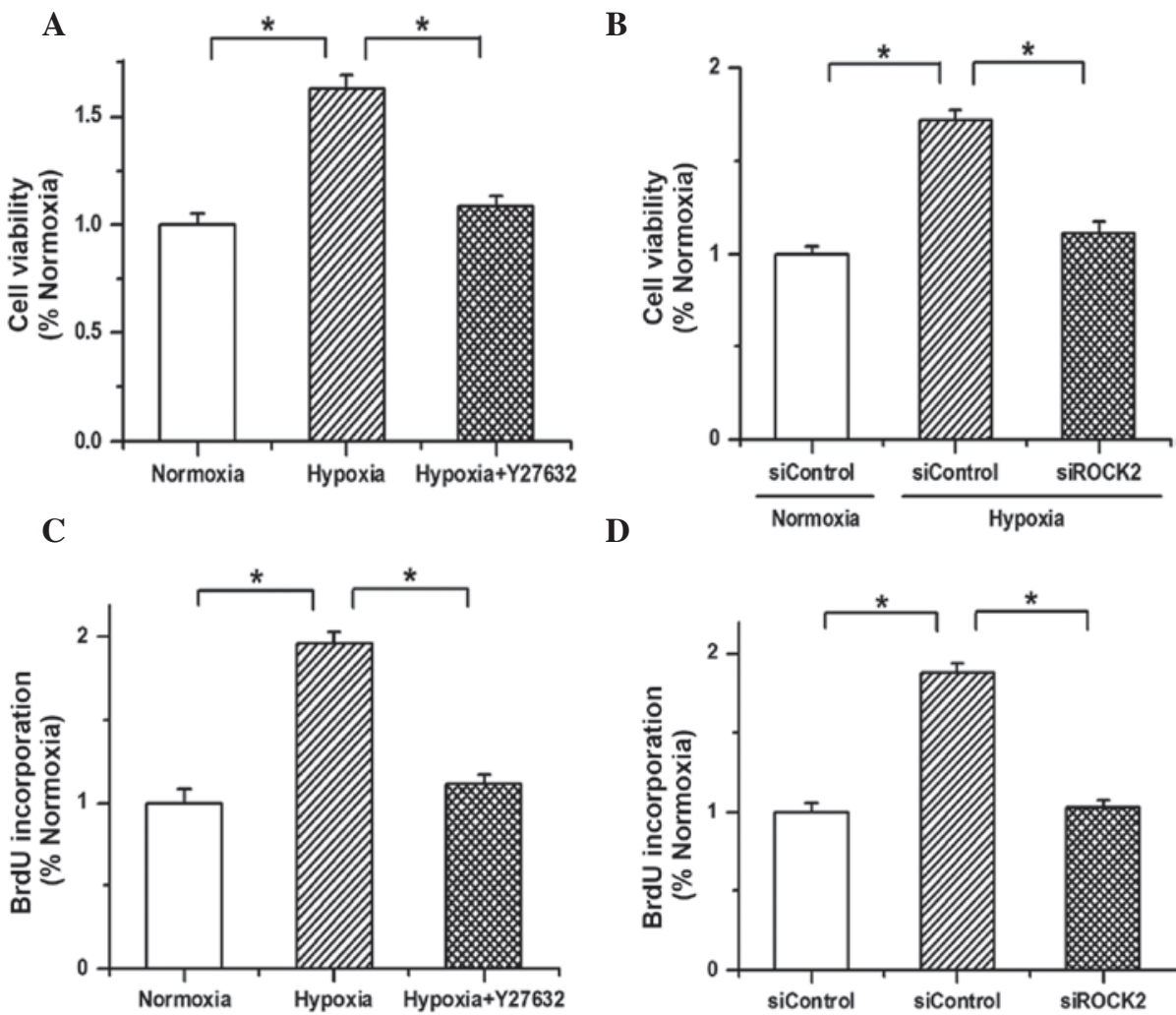

D

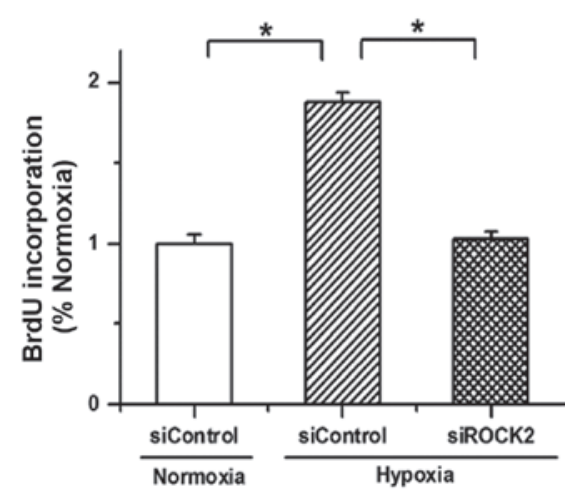

Figure 3. Hypoxia promotes the proliferation of PAECs through the ROCK2 pathway. (A) The results of an MTT assay showed that the cell viability was enhanced by hypoxia treatment, but the promotion effects of hypoxia on cell growth were weakened by Y27632. (B) The increased cell viability of PAECs induced by hypoxia was attenuated by ROCK2 siRNA (siROCK2). (C) Hypoxia enhanced the incorporation of BrdU in PAECs, which was repressed by Y27632. (D) The proliferation of PAECs promoted by hypoxia was attenuated by siROCK2. All values are presented as the mean \pm standard error of the mean from three or more independent batches of cells. "P<0.05, as detected by one-way analysis of variance and Dunnett's test. PAECs, pulmonary arterial endothelial cells; ROCK2, Rho-kinase $\alpha$; MTT, 3-(4,5-dimethylthiazol-2-yl)-2,5-diphenyl-tetrazolium bromide; siRNA, small interfering RNA; siControl, control siRNA; BrdU, 5-bromo-2'-deoxyuridine.

Statistical analysis. Composite data are expressed as mean \pm standard error of the mean. Statistical analysis was performed with Student's t-test or one-way analysis of variance followed by Dunnett's test where appropriate. $\mathrm{P}<0.05$ was considered to indicate a statistically significant difference.

\section{Results}

Hypoxia increases the activity and expression of ROCK2 in PAECs. First, whether the ROCK2 pathway is activated under hypoxic condition in PAECs was examined. As shown in Fig. 1A and B, hypoxia induced the mRNA and protein expression of ROCK2 in PAECs. Moreover, as the phosphorylation of MYPT1, a downstream target of ROCK, reflects ROCK2 activity, the amounts of p-Thr853 MYPT1 and total MYPT1 were determined. The western blotting results showed that the phosphorylation of MYPT1 was significantly increased in PAECs following stimulation with hypoxia compared with the normoxic group (Fig. 1C). These results indicate that hypoxia activates the ROCK 2 pathway by upregulating ROCK 2 expression and enhancing its activity in PAECs.

ROCK2 activity is blocked by Y27632 and ROCK2 siRNA significantly represses the expression of ROCK2. To demonstrate the important roles of ROCK2 in PAECs treated with hypoxia, Y27632 (an inhibitor of ROCK) was used to block the activation of the ROCK pathway. It was found that the phosphorylation of MYPT1 induced by hypoxia was clearly decreased by incubation with $1 \mu \mathrm{M}$ Y27632 (Fig. 2A). As possible nonspecific inhibition by the chemical inhibitor may occur, specific siRNA was also used to silence the gene expression of ROCK2 in PAECs. The expression of ROCK2 was examined by RT-qPCR and western blotting to determine the knockdown efficiency. As shown in Fig. 2B and C, the mRNA and protein expression of ROCK2 was significantly reduced by siROCK 2 .

Hypoxia promotes the proliferation of PAECs through the ROCK2 pathway. The MTT assay was conducted to determine whether the effects of hypoxia on PAEC proliferation are dependent on the ROCK2 pathway. It was found that cell viability was increased by hypoxia treatment, but the promotive effects of hypoxia on cell growth were weakened by Y27632 (Fig. 3A; $\mathrm{n}=3, \mathrm{P}<0.05$ ). Similar results were obtained after knocking down the expression of ROCK2 with siROCK2; the cell viability increased by hypoxia was repressed (Fig. 3B; n=3, P<0.05). Furthermore, the results of the BrdU incorporation assay showed that hypoxia increased the incorporation of BrdU into PAECs, which was repressed by Y27632 or the ROCK2 siRNA (Fig. 3C and D; $n=3$, $\mathrm{P}<0.05)$. These results indicate that the proliferation of PAECs induced by hypoxia is mediated by ROCK 2 . 
A

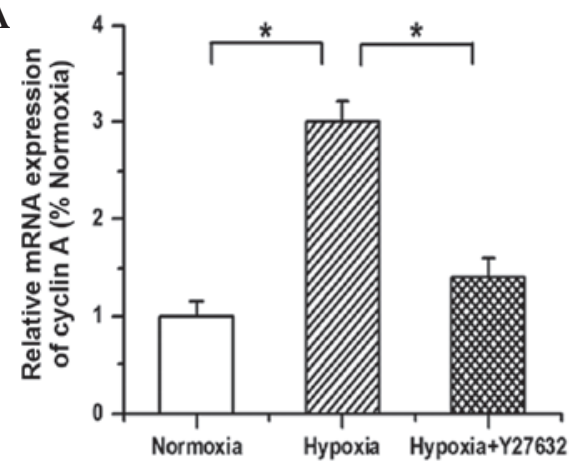

C

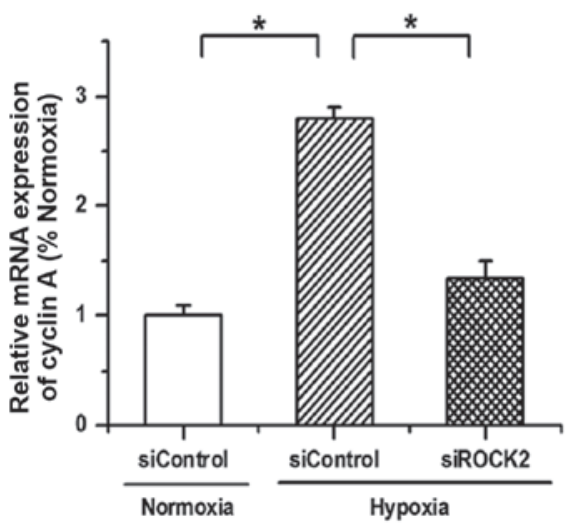

B

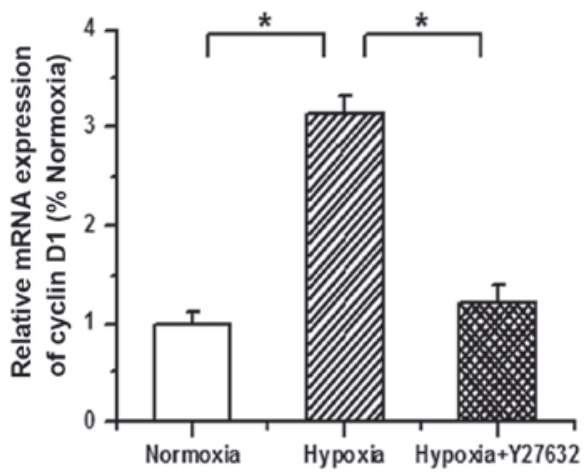

D

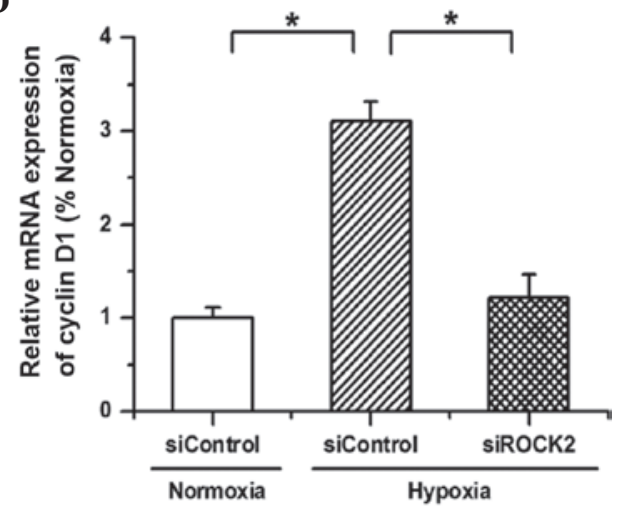

Figure 4. Hypoxia regulates the mRNA expression of cell cycle proteins in pulmonary arterial endothelial cells to advance cell cycle progression, which is weakened by ROCK2 siRNA (siROCK2) or Y27632. (A) The mRNA expression of cyclin A upregulated by hypoxia was inhibited by Y27632. (B) The mRNA expression of cyclin D1 induced by hypoxia was reversed by Y27632. (C) Silencing ROCK2 expression with siRNA weakened the promotion effects of hypoxia on cyclin A expression. (D) The effects of hypoxia on cyclin D1 expression were attenuated by siROCK2. All values are presented as the mean \pm standard error of the mean from three or more independent batches of cells. "P $<0.05$, as detected by one-way analysis of variance and Dunnett's test. ROCK2, Rho-kinase $\alpha$; siRNA, small interfering RNA; siControl, control siRNA.

Hypoxia regulates the expression of cell cycle proteins to advance the cell cycle and its effects are attenuated by siROCK2 or Y27632. Since the aforementioned results have demonstrated that hypoxia promotes the proliferation of PAECs via ROCK2, the expression of cell-cycle regulatory proteins was then detected to verify this conclusion. As shown in Fig. 4, the mRNA expression of cyclin A and cyclin D1 was upregulated by hypoxia, and the promotive effects of hypoxia on the expression of cyclin A and cyclin D1 were reversed after either blocking the ROCK2 pathway with Y27632 or silencing the ROCK2 expression with siRNA. These results indicate that hypoxia promotes the proliferation of PAECs by regulating the expression of cell cycle proteins to advance cell cycle progression.

\section{Discussion}

Endothelial dysfunction is acknowledged as a common pathological feature of PAH, which results from the formation of plexiform lesions by the disordered overgrowth of endothelial cells (14). Usually, inhibiting apoptosis or promoting proliferation leads to the overgrowth of PAECs. The overgrowth of pulmonary vascular endothelial cells is an important feature of $\mathrm{PAH}$, and the proliferated endothelial cells form tumorlets and plexiform lesions, which can obliterate medium-sized arteries and raise the pulmonary vascular pressure in the development of PAH $(14,15)$. Therefore, it is necessary to determine the regulatory mechanism of proliferation in PAECs. The results of the present study provide new evidence that ROCK2 mediates the proliferation of PAECs induced by hypoxia.

A major finding of the present study is that ROCK2 participates in the hypoxia-induced hypertrophy of the pulmonary artery intima. There are two basic pathological changes in the development of PAH: One is sustained pulmonary vasoconstriction and the other is vascular remodeling. Previous studies have demonstrated that the development of PAH induced by monocrotaline or hypoxia is attenuated following a long-term treatment with fasudil (an isoform-nonspecific ROCK inhibitor) $(16,17)$. Furthermore, increased ROCK activation has been observed in patients with idiopathic PAH (18). Moreover, it has been reported that the Rho/ROCK pathway is involved in the regulation of pulmonary vasoconstriction $(18,19)$. This evidence indicates that the ROCK pathway serves as an important signal transduction pathway in the progression of PAH. However, the majority of studies have focused on the effects of ROCK on the pulmonary vascular media (particularly pulmonary artery smooth muscle cells) in blood vessel remodeling. Whether the ROCK pathway is involved in the mechanism by which hypoxia induces intimal hypertrophy remains unknown. As the major component of vessel intima, PAECs were investigated used in the present study. It was found that the activity and expression of ROCK 2 were both increased by hypoxia 
in cultured PAECs. Moreover, hypoxia promoted the growth and proliferation of PAECs through activation of the ROCK2 signaling pathway. These results provide direct evidence that the effects of hypoxia on the overgrowth of PAECs are mediated by ROCK 2 .

In addition, cyclin D1 and cyclin A are closely associated with progression of the cell cycle. The increased expression of cyclin D1 and cyclin A causes an increased number of cells to transition from the G0/G1 phase to the $\mathrm{S}$ phase, and cell proliferation is significantly enhanced (20). In the present study, hypoxia induced the expression of cyclin D1 and cyclin A, but the ROCK inhibitor Y27632 and ROCK2 siRNA each attenuated the effects of hypoxia on the expression of cell cycle proteins. These results indicate that hypoxia regulates the expression of cell cycle proteins to advance cell cycle progression and promotes cell proliferation via ROCK2.

In conclusion, the results of the present study indicate that hypoxia advances the progression of the cell cycle and promotes cell proliferation through activating the ROCK2 pathway in PAECs. This finding may highlight an important mechanism in the development of $\mathrm{PAH}$, and thus may suggest a novel target for improving the treatment of $\mathrm{PAH}$ in the future.

\section{References}

1. Humbert M, Sitbon O, Chaouat A, Bertocchi M, Habib G, Gressin V, Yaïci A, Weitzenblum E, Cordier JF, Chabot F, et al: Survival in patients with idiopathic, familial and anorexigen-associated pulmonary arterial hypertension in the modern management era. Circulation 122: 156-163, 2010.

2. Fukumoto $\mathrm{Y}$ and Shimokawa H: Recent progress in the management of pulmonary hypertension. Circ J 75: 1801-1810, 2011.

3. Morrell NW, Adnot S, Archer SL, Dupuis J, Jones PL, MacLean MR, McMurtry IF, Stenmark KR, Thistlethwaite PA, Weissmann $\mathrm{N}$, et al: Cellular and molecular basis of pulmonary arterial hypertension. J Am Coll Cardiol 54 (Suppl 1): S20-S31, 2009.

4. Shimokawa H and Rashid M: Development of Rho-kinase inhibitors for cardiovascular medicine. Trends Pharmacol Sci 28: 296-302, 2007.

5. Satoh K, Fukumoto Y and Shimokawa H: Rho-kinase: Important new therapeutic target in cardiovascular diseases. Am J Physiol Heart Circ Physiol 301: H287-H296, 2011.

6. Nakagawa O, Fujisawa K, Ishizaki T, Saito Y, Nakao K and Narumiya S: ROCK-I and ROCK-II, two isoforms of rho-associated coiled-coil forming protein serine/threonine kinase in mice. FEBS Lett 392: 189-193, 1996.
7. Chevrier V, Piel M, Collomb N, Saoudi Y, Frank R, Paintrand M, Narumiya S, Bornens M and Job D: The rho-associated protein kinase p160ROCK is required for centrosome positioning. J Cell Biol 157: 807-817, 2002

8. Wei L, Roberts W, Wang L, Yamada M, Zhang S, Zhao Z, Rivkees SA, Schwartz RJ and Imanaka-Yoshida K: Rho kinases play an obligatory role in vertebrate embryonic organogenesis. Development 128: 2953-2962, 2001.

9. Shimizu Y, Thumkeo D, Keel J, Ishizaki T, Oshima H, Oshima M, Noda Y, Matsumura F, Taketo MM and Narumiya S: ROCK-I regulates closure of the eyelids and ventral body wall by inducing assembly of actomyosin bundles. J Cell Biol 168: 941-953, 2005.

10. Thumkeo D, Keel J, Ishizaki T, Hirose M, Nonomura K, Oshima H, Oshima M, Taketo MM and Narumiya S: Targeted disruption of the mouse rho-associated kinase 2 gene results in intrauterine growth retardation and fetal death. Mol Cell Biol 23: 5043-5055, 2003.

11. Medhora M, Chen Y, Gruenloh S, Harland D, Bodiga S, Zielonka J, Gebremedhin D, Gao Y, Falck JR, Anjaiah S and Jacobs ER: 20-HETE increases superoxide production and activates NAPDH oxidase in pulmonary artery endothelial cells. Am J Physiol Lung Cell Mol Physiol 294: L902-L911, 2008.

12. Ma J, Zhang L, Li S, Liu S, Ma C, Li W, Falck JR, Manthati VL, Reddy DS, Medhora M, et al: 8,9-Epoxyeicosatrienoic acid analog protects pulmonary artery smooth muscle cells from apoptosis via ROCK pathway. Exp Cell Res 316: 2340-2353, 2010.

13. Livak KJ and Schmittgen TD: Analysis of relative gene expression data using real-time quantitative PCR and the $2^{-\Delta \Delta C t}$ method. Methods 25: 402-408, 2001.

14. Budhiraja R, Tuder RM and Hassoun PM: Endothelial dysfunction in pulmonary hypertension. Circulation 109: 159-165, 2004.

15. Pidgeon GP, Tamosiuniene R, Chen G, Leonard I, Belton O, Bradford A and Fitzgerald DJ: Intravascular thrombosis after hypoxia-induced pulmonary hypertension: Regulation by cyclooxygenase-2. Circulation 110: 2701-2707, 2004.

16. Abe K, Tawara S, Oi K, Hizume T, Uwatoku T, Fukumoto Y, Kaibuchi $\mathrm{K}$ and Shimokawa H: Long-term inhibition of Rho-kinase ameliorates hypoxia-induced pulmonary hypertension in mice. J Cardiovasc Pharmacol 48: 280-285, 2006.

17. Abe K, Shimokawa H, Morikawa K, Uwatoku T, Oi K, Matsumoto Y, Hattori T, Nakashima Y, Kaibuchi K, Sueishi K and Takeshit A: Long-term treatment with a Rho-kinase inhibitor improves monocrotaline-induced fatal pulmonary hypertension in rats. Circ Res 94: 385-393, 2004.

18. Do e Z, Fukumoto Y, Takaki A, Tawara S, Ohashi J, Nakano M, Tada T, Saji K, Sugimura K, Fujita H, et al: Evidence for Rho-kinase activation in patients with pulmonary arterial hypertension. Circ J 73: 1731-1739, 2009.

19. Oka M, Fagan KA, Jones PL and McMurtry IF: Therapeutic potential of RhoA/Rho kinase inhibitors in pulmonary hypertension. Br J Pharmacol 155: 444-454, 2008.

20. Katula KS, Wright KL, Paul H, Surman DR, Nuckolls FJ, Smith JW, Ting JP, Yates J and Cogswell JP: Cyclin-dependent kinase activation and S-phase induction of the cyclin B1 gene are linked through the CCAAT elements. Cell Growth Differ 8: 811-820, 1997. 